\title{
Evaluación de la Remoción de Nitrógeno, Fósforo y Sulfuros en Agua Residual Doméstica, Utilizando Phragmites australis en Bioreactores
}

\author{
Sandra N. Correa-Torres, Yolanda Gamarra, Andrés A. Salazar, Natalia M. Pitta \\ Facultad de Ingeniería Ambiental, Universidad Pontificia Bolivariana - seccional Bucaramanga. Autopista \\ a Piedecuesta Km 7, Bucaramanga-Colombia (e-mail: sandra.correa@upb.edu.co)
}

Recibido Abr. 30, 2015; Aceptado Jun. 8, 2015; Versión final Sep. 1, 2015, Publicado Dic. 2015

\begin{abstract}
Resumen
En el presente estudio se evaluó la remoción de nitrógeno, fósforo y sulfuros en un agua residual doméstica mediante fitorremediación utilizando Phragmites australis en bioreactores a escala de laboratorio. Se determinó el estudio las condiciones hidráulicas en reactores analizando las variables de relación geométrica largo:ancho (4:1 y 1:4) y usando un medio filtrante (grava/arena). Adicionalmente se evaluó el crecimiento de $P$. australis en los reactores y se analizó los porcentajes de remoción para cada configuración. Los resultados hidráulicos indicaron que los reactores con configuración 4:1 presentan un comportamiento de flujo a pistón contrario a los reactores en configuración 1:4 que presentan una mayor tendencia de flujo mezclado. Los valores de remoción de contaminantes indican mayor remoción de fósforo (60 a $90 \%$ ) en todos los medios filtrantes de los bioreactores y menor remoción de nitrógeno (13\%). Los sulfuros fueron removidos en un $20 \%$. El mayor porcentaje de remoción se presentó en los reactores de grava 4:1, siendo esta la mejor condición para optimizar la extracción usando $P$. australis.
\end{abstract}

Palabras clave: condiciones hidráulicas, configuración geométrica, Phragmites australis, remoción de nutrientes

\section{Evaluation of Nitrogen, Phosphorus and Sulphur Removal in Domestic Wastewater, using Phragmites australis in bioreactors}

\begin{abstract}
In the present study, nitrogen, phosphorus and sulfur removal was evaluated in domestic wastewater by phytoremediation using Phragmites australis in laboratory scale bioreactors. The hydraulic conditions in the reactor were determined by analyzing the geometric variables long:width ratio (4:1 and 1:4) and using a filter medium (gravel / sand). Additionally, the $P$. australis growth was evaluated and removal percentages for each configuration were analyzed. The results indicated that water reactor with setting 4:1 present piston flow while configuration 1:4 present a behavior similar to mixed flow. Values of contaminant removal indicate higher phosphorus removal (60 to 90\%) in all reactors and lower nitrogen removal (13\%). The sulfides removal was $20 \%$. The highest percentage of removal was presented in reactors gravel $4: 1$ being the best condition to optimize the extraction of $P$. australis.
\end{abstract}

Keywords: hydraulic conditions; geometric conditions; Phragmites australis; nutrient removal 


\section{INTRODUCCIÓN}

La utilización de humedales como tratamiento de agua residual se ha desarrollado e investigado ampliamente a nivel internacional desde 1970, en Estados Unidos y Europa, debido a la alta eficiencia de remoción reportada por los mismos, así como su bajo costo de construcción y mantenimiento, en comparación con los sistemas convencionales empleados en Latinoamérica (Vera, 2014; Valencia, 2010; Kadlec 2009) En sistemas de aguas residuales desarrollados a nivel internacional se han usado las plantas fitoremediadoras de sustancias presentes en aguas, entre ellas están P. australis. (Gonzalias, 2007; Kalipci, 2011; González, 2014). Esta planta ha sido ampliamente utilizada para la remoción de nitrógeno y fosforo, obteniendo resultados de remoción del 88 y 99\% respectivamente (Wiessner, 2010).

En Colombia, las técnicas de tratamiento de aguas residuales empleadas actualmente, presentan inconvenientes operativos y requerimientos excesivos del sistema, lo cual exige la modificación e implementación de procesos, sistemas o alternativas correctivas, para incrementar la eficiencia de remoción y cumplir la normativa ambiental (Valencia, 2010; Passarini, 2012; Rodríguez, 2008). En el caso de Colombia la planta $P$. australis ha sido poco empleada en la remoción de nitrógeno y fósforo y no ha sido estudiada en la remoción de sulfuros en aguas residuales domésticas. $P$. australis que es una macrófita acuática, cosmopolita, es considerada una excelente alternativa en procesos de remoción de nitrógeno, fósforo, sulfuros y puede aplicarse en una etapa terciaria de tratamiento de aguas residuales (Vymazal, 2008; Carlson, 2014). $P$. australis presenta una tolerancia a DQO hasta de $128 \mathrm{mg} / \mathrm{L}$, resiste temperaturas entre $12-25^{\circ} \mathrm{C}$, soporta salinidadades máxima de 45 ppt en aguas y resiste a pH aproximado de 2.0 a 8.0. (González 2014, Espinosa, 2014; Ayaz, 2008).

En el presente estudio se utiliza el efluente del tratamiento secundario de la planta de tratamiento de agua residual (PTAR) de un conjunto residencial localizado en el área metropolitana de Bucaramanga-Colombia, el cual cuenta con una población aproximada de 280 habitantes. La PTAR está compuesta por un sistema preliminar de rejillado grueso y fino; tratamiento primario por medio de un sedimentador con lechos de secado de lodos; y el secundario consiste en un filtro biológico con aireación extendida. Por tanto, el efluente de la PTAR enunciada presenta un bajo contenido de materia orgánica y sólidos (suspendidos y disueltos), lo cual favorece la operación del sistema con flujo subsuperficial (humedal artificial) a manera de tratamiento terciario para la remoción de nutrientes. En el agua a tratar las concentraciones iniciales de nitrógeno $(3.2 \mathrm{mg} / \mathrm{L}$ de $\mathrm{NH}_{3}$ y $0.05 \mathrm{mg} / \mathrm{L}$ de $\mathrm{N}$ orgánico) y fósforo (1.35 mg/L de fósforo orgánico) son bajas y aptas para cumplir la normativa, pero los sulfuros $(0.5 \mathrm{mg} / \mathrm{L}$ sulfuros) se encuentran en altos valores.

En este caso específico, la implementación de tratamientos convencionales para la disminución de cargas contaminantes en agua residual doméstica incluye inconvenientes operativos en el tratamiento terciario, provenientes de la presencia de nitrógeno, fósforo y por la presencia de sulfuros. Con el fin de solucionar esta problemática y presentar en Colombia una posible solución a la remoción de sulfuros, en el presente estudio se implementó una técnica de fitorremediación utilizando la planta $P$. australis para un tratamiento con flujo subsuperficial en bioreactores a escala de laboratorio. Se establecieron tres objetivos: el primero corresponde a la determinación de las condiciones hidráulicas optimas de los bioreactores para la remoción de nitrógeno, fósforo y sulfuros en aguas residuales domésticas, utilizando $P$. australis, mediante la evaluación de variables como la relación geométrica largo:ancho $(4: 1$ y $1: 4)$ y usando un medio filtrante de grava,arena y un control sin medio. Adicionalmente se evaluó la influencia de estos en la eficiencia hidráulica del sistema propuesto. Como segundo objetivo se determinó el crecimiento de los individuos seleccionados de $P$. australis y como tercer objetivo se determinó los porcentajes de remoción de nitrógeno, fósforo y sulfuros en una configuración hidráulica y en un medio filtrante adecuado.

\section{MATERIALES Y MÉTODOS}

Los experimentos fueron realizados en biorreactores de láminas de acero inoxidable con dimensiones de 100 $\mathrm{cm}$ de largo por 25 de ancho a escala laboratorio para el tratamiento terciario con flujo subsuperficial de un agua doméstica. En el diseño y construcción de los biorreactores fueron evaluadas las variables de configuración geométrica 4:1 y 1:4 (largo: ancho) y el medio filtrante (grava y arena). La experimentación se llevó a cabo usando seis biorreactores, tres corresponden a la configuración 4:1 y tres a la configuración 1:4. Para cada configuración se analizó un bioreactor con grava y un bioreactor con arena y un bioreactor control, con un tiempo de retención hidráulico (TRH) de 30 horas, ilustrados en la Figura 1. 


\section{Parámetros hidráulicos}

Con el fin de determinar el comportamiento hidráulico en los bioreactores, se realizó la obtención de una curva distribución de tiempos de residencia (DTR), donde se empleó una solución de cloruro de potasio (KCl) a concentración de $110 \mathrm{~g} \mathrm{~L}^{-1}$ como trazador (Mastrocicco, 2011; Al-Dahhan, 2006, Naderi Nasrabadi M. 2014). El pulso inicial fue de $50 \mathrm{ml}$ y se monitoreó la conductividad en el efluente de cada uno de los bioreactores, en intervalos de treinta minutos. En el efluente de cada bioreactor se tomaron tres muestras del agua residual desde la inyección del trazador, durante un periodo de tiempo equivalente a tres veces el tiempo de retención hidráulico-teórico TRH-teórico (90 horas).

Para estimar el tiempo de residencia del fluido en el bioreactor, se aplicaron criterios nominales tales como la estimación del Índice de Cortos Circuitos, Índice de Morril, tasa de recuperación del trazador y eficiencia hidráulica. Adicionalmente se utilizaron los métodos de Wolf-Resnick y análisis por curva de tendencia con el fin de establecer el tipo de flujo y la presencia de cortos circuitos y zonas muertas dentro de cada configuración de los bioreactores (Zataray, 2006; Vargas, 2005; Sabas, 2011). Para la evaluación estadística de la tendencia de los datos hidráulicos, se aplicó un análisis de varianza (ANOVA) y una prueba de Scheffé. En este diseño se empleó un Factorial Completo $2^{k}$, estableciendo como factores la relación geométrica $(4: 1$ y $1: 4)$ y como variables los medios filtrantes (arena, grava y control).

(a)

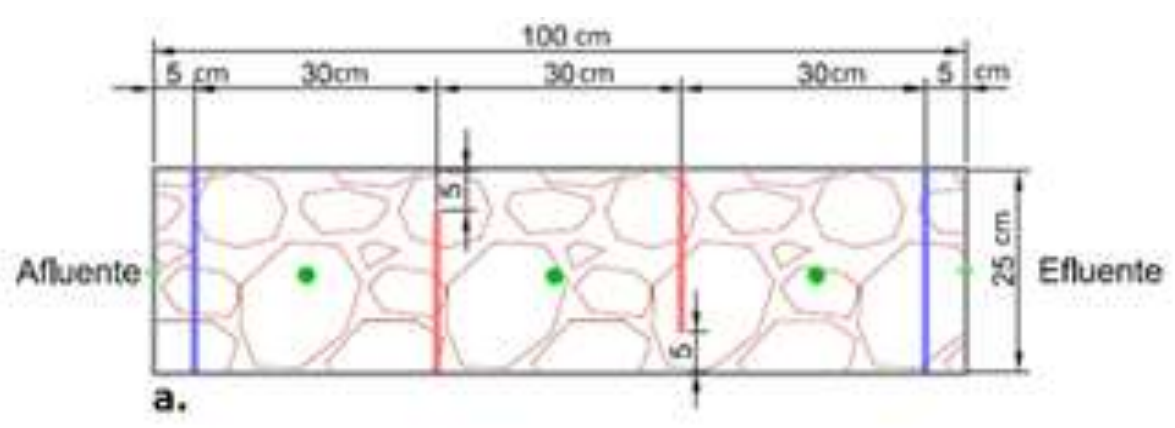

(b)

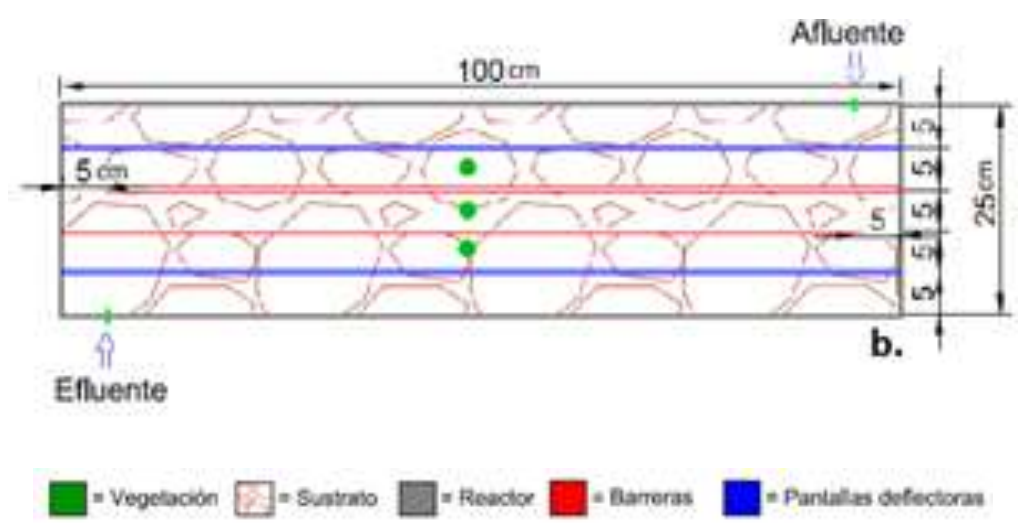

Fig. 1: Diseño de los bioreactores a escala de laboratorio con vista superior y foto de los bioreactores: a) Configuración 4:1 (Largo:Ancho), b) Configuración 1:4 (Largo:Ancho).

\section{Análisis de remoción de nitrógeno, fósforo y sulfuros en bioreactores}

Con los parámetros hidráulicos seleccionados se colocó en cada bioreactor la especie vegetativa $P$. australis seleccionada como objeto de estudio fundamentada en su capacidad para asimilar nutrientes (Vymazal, 2008; Gonzalias, 2007). La adaptación, crecimiento y reproducción de las plántulas de $P$. australis se realizó con agua residual doméstica durante un periodo de seis meses donde se seleccionaron tres individuos para cada bioreactor. El funcionamiento del sistema se inició tomando muestras en el suministro y en el efluente de cada reactor en intervalos de 30 horas, durante un periodo de tiempo equivalente a seis veces el THR-teórico (180 horas). 
Para evaluar la concentración de nitrógeno total en las muestras del afluente y efluente de cada bioreactor, se aplicó la técnica de digestión por Kjeldahl. La concentración de fósforo se determinó por digestión ácida por análisis Ultravioleta Visible. La concentración de sulfuros se determinó usando el método por electrodo de ion selectivo, relacionando la actividad del ion sulfuro. Los análisis químicos de los respectivos elementos se realizaron en el Laboratorio de Análisis Químico de Aguas Residuales de la Universidad Pontificia Bolivariana-Seccional Bucaramanga, acreditado por el Instituto de Hidrología, Meteorología y Estudios Ambientales de Colombia (IDEAM).

\section{Crecimiento vegetal}

Los individuos de $P$. australis seleccionados y adaptados fueron trasplantados a los bioreactores, donde se implementó un sistema de flujo continuo para el agua residual doméstica a analizar. En cada bioreactor se ubicaron tres plantas de $P$. australis con características de crecimiento homogéneas de $1 \mathrm{~m}$ y adaptadas en un periodo de tiempo de seis meses al agua residual. Después de la adaptación las plantas fueron colocadas a una distancia de $15 \mathrm{~cm}$ cada una en la configuración $4: 1$ y de $5 \mathrm{~cm}$ en la configuración 1:4.

Los ejemplares de $P$. australis seleccionados para su uso en los reactores se ubicaron de forma escalonada dentro de los mismos, siendo $60 \mathrm{~cm}$ la profundidad del más cercano al efluente, $70 \mathrm{~cm}$ en el siguiente y 80 $\mathrm{cm}$ en el cercano al afluente, para las dos configuraciones de los bioreactores. Para evaluar el crecimiento vegetal se determinó el número de hojas, longitud de la raíz y del tallo de cada individuo, con la cual se comparó la medición inicial con la obtenida después de dos semanas.

\section{RESULTADOS Y DISCUSIÓN}

\section{Curvas de Distribución}

Las curvas de distribución de tiempo de residencia (DTR) en las configuraciones 1:4 y 4:1 fueron obtenidas por el estudio del tiempo de residencia de la solución del trazador y son presentadas en la Figura 2. Las curvas de DTR exhiben mayor homogeneidad hidráulica en los reactores de configuración 1:4. En la configuración 4:1 con grava, se observa una mayor variabilidad, lo cual está relacionado con la distancia entre barreras y sus consecuentes porcentajes altos de zonas muertas. El reactor de arena configuración 4:1 se observa un comportamiento lineal, sin distribución gaussiana, favoreciendo la acumulación del trazador en las zonas muertas.

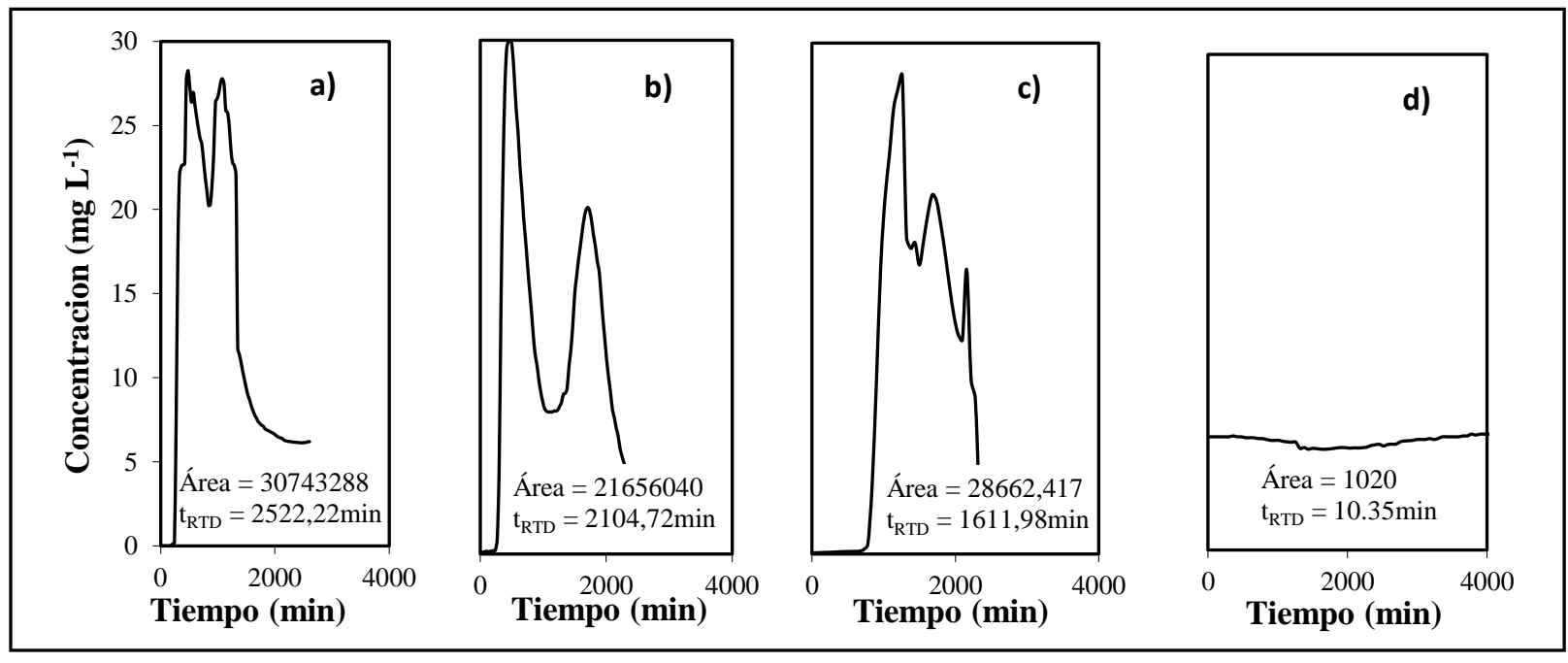

Fig. 2: Curva DTR para los bioreactores de Configuración 1:4: a) arena, b) grava; los bioreactores de Configuración 4:1: c) arena, d) grava

Los criterios hidráulicos matemáticos fueron aplicados a las curvas DTR, los resultados son presentados en la Tabla 1, permitiendo definir los mejores comportamientos hidráulicos en los bioreactores de las dos configuraciones. Los resultados evidenciaron que los bioreactores control de las dos configuraciones geométricas presentan mayores deficiencias hidráulicas (zonas muertas y cortos circuitos) en relación con los reactores de grava y arena. 
Los resultados de los criterios aplicados indican que el bioreactor con grava de la configuración 4:1 es hidráulicamente superior en esta configuración a las otras unidades de tratamiento, destacando de esto la eficiencia hidráulica (69-88\%) según cálculos utilizando ecuaciones de Persson y Tacktson y el valor más bajo en porcentaje de zonas muertas (15,8\%). Adicionalmente el reactor grava configuración 4:1 presentan una curva hidráulica con una distribución más uniforme en el tiempo, lo que demuestra la eficiencia de los bafles para definir el recorrido dentro del reactor y del medio filtrante para homogenizar el flujo e incrementar el TRH real (en relación al teórico). La tabla 1 demostró que para la configuración 4:1 el reactor grava es el de mejor comportamiento hidráulico determinando un flujo pistón.

Tabla 1: Criterios hidráulicos aplicados a las curvas DTR

\begin{tabular}{|c|c|c|c|c|c|c|}
\hline \multirow[b]{2}{*}{ Criterio } & \multicolumn{3}{|c|}{ Configuración 1:4 } & \multicolumn{3}{|c|}{ Configuración 4:1 } \\
\hline & Grava & Arena & Control & Grava & Arena & Control \\
\hline TRH real (h) & 20.12 & 18.34 & 19.55 & 26.86 & 47.05 & 9.51 \\
\hline Índice de Morril & 4.57 & 4.30 & 2.99 & 2.12 & 9.25 & 2.21 \\
\hline Eficiencia hidráulica (Tacktson) & 66.4 & 62.5 & 64.4 & 88.6 & 160.3 & 31.3 \\
\hline Eficiencia hidráulica (Persson) & 39.8 & 43.6 & 115.1 & 69.3 & 269.2 & 23.0 \\
\hline$\%$ Trazador recuperado & 53.6 & 61.4 & 46.5 & 62.6 & 82.0 & 30.3 \\
\hline Wolf-Resnick p (F. Pistón) & 29.5 & 21.5 & 63.9 & 59.4 & 0.00 & 68.1 \\
\hline Wolf-Resnick m (\% Z. Muertas) & 34.3 & 30.1 & 43.5 & 15.8 & ------- & 75.5 \\
\hline Wolf-Resnick M (\% F. Mezclado) & 70.5 & 78.5 & 36.1 & 40.6 & 1.00 & 31.9 \\
\hline Curva de tendencia Ic=ti/to & C.Circuito & C.Circuito & C.Circuito & Normal & C.Circuito & C.Circuito \\
\hline Curva de tendencia tm/to & C.Circuito & C.Circuito & C.Circuito & C.Circuito & Z. Muertas & C.Circuito \\
\hline Curva de tendencia tc/to & N.A & N.A & N.A & N.A & N.A & N.A \\
\hline Curva de tendencia tb/to & F. E. I. & F. E. I. & F. E.I. & F. E. I. & N.A & F. E.I \\
\hline $\begin{array}{l}\text { Curva de tendencia } \\
\mathrm{e}=((\mathrm{tf}-\mathrm{tp})(\mathrm{tp}-\mathrm{ti})) / \text { to }\end{array}$ & F.M.I. & F. Pistón & F. Pistón & F. Pistón & F. Pistón & F. Pistón \\
\hline
\end{tabular}

F.E.I: Flujo Estable Ideal; F.M.I: Flujo Mezclado Ideal; N.A: no aplica

El comportamiento de los bioreactores en la configuracion 1:4 presenta deficiencias hidráulicas por la presencia de cortos circuitos en todos los medios filtrantes. Resaltando el bioreactor de arena con una eficiencia hidráulica entre el $43,6 \%$ y el $62,5 \%$ (Persson y Tackston respectivamente), un porcentaje de zonas muertas del $30,1 \%$ menor en comparación a otros medios y un comportamiento de $78,5 \%$ con tendencia a flujo mezclado y $21,5 \%$ de flujo a pistón y un porcentaje de recuperación del trazador del $61,4 \%$.Debido a la variabilidad de los resultados y para poder establecer la mejor configuración se realizó el análisis por ANOVA.

El análisis realizado por ANOVA para la evaluación estadística de la tendencia de los datos hidráulicos, establecidos para cada medio filtrante en relación a la configuración, con un factor $2^{2}$ y con un nivel de confianza del 95\%, presentó diferencias significativas en la configuración geométrica 1:4 y 4:1. Estos resultados indicaron que existen diferencias entre las configuraciones de los bioreactores de las medias cuadráticas y su respectivo factor $F(>>1)$, lo cual permite rechazar la hipótesis nula de igualdad de la configuración de los bioreactores y proceder con los análisis de Dunnet y Sheffé. Estas pruebas post hoc permitieron determinar la variabilidad entre los reactores en términos de medio filtrante para cada configuración y en cuanto a la tendencia de las curvas DTR.

Para la configuración 4:1 los resultados del análisis de las pruebas de Dunnet y de Scheffé demostraron que mediante el análisis del intervalo en que se ubican los límites inferior y superior, indicó que existe una tendencia similar entre los bioreactores de control y arena, pero no con reactor de grava donde el comportamiento es diferente. En la configuración 1:4 el valor resultante con base en las dos pruebas estadísticas aplicadas (Scheffé y t de Dunnet) resultó viable establecer que los reactores con arena y grava difieren en términos de concentración promedio del reactor control, pero el comportamiento hidráulico de los reactores grava y arena se asemeja.

El análisis estadístico indica que para ambas configuraciones el comportamiento hidráulico es diferente en cada bioreactor. Para la configuración 4:1 el medio filtrante con grava tuvo el mejor comportamiento hidráulico en comparación con la configuración 1:4 los reactores grava y arena tienden a tener un comportamiento semejante. 


\section{Remoción de nitrógeno, fósforo y sulfuros}

La remoción de los contaminantes por medio de $P$. asutralis fue influenciada por la configuración de los bioreactores. En la Figura 3, se observó que el nitrógeno presentó bajos porcentajes de remoción en las dos configuraciones 1:4 y 4:1. El nitrógeno presente en el agua residual doméstica después de un tratamiento secundario y con la aplicación de $P$. asutralis como tratamiento terciario obtuvo bajas concentraciones por debajo de los límites de detección de la técnica analítica, presentando resultados no cuantificables en la configuración 1:4. En literatura reporta porcentajes de remoción de 88 y 45 (Wathugala, 1986 y Lee, 2009) para nitrógeno total en reactores de flujo subsuperficial, se evidencia que los datos obtenidos difieren en gran magnitud de los reportados en la literatura, esto se deber a las variaciones ambientales y a la duración de la experimentación que es menor a la presentada en la literatura.
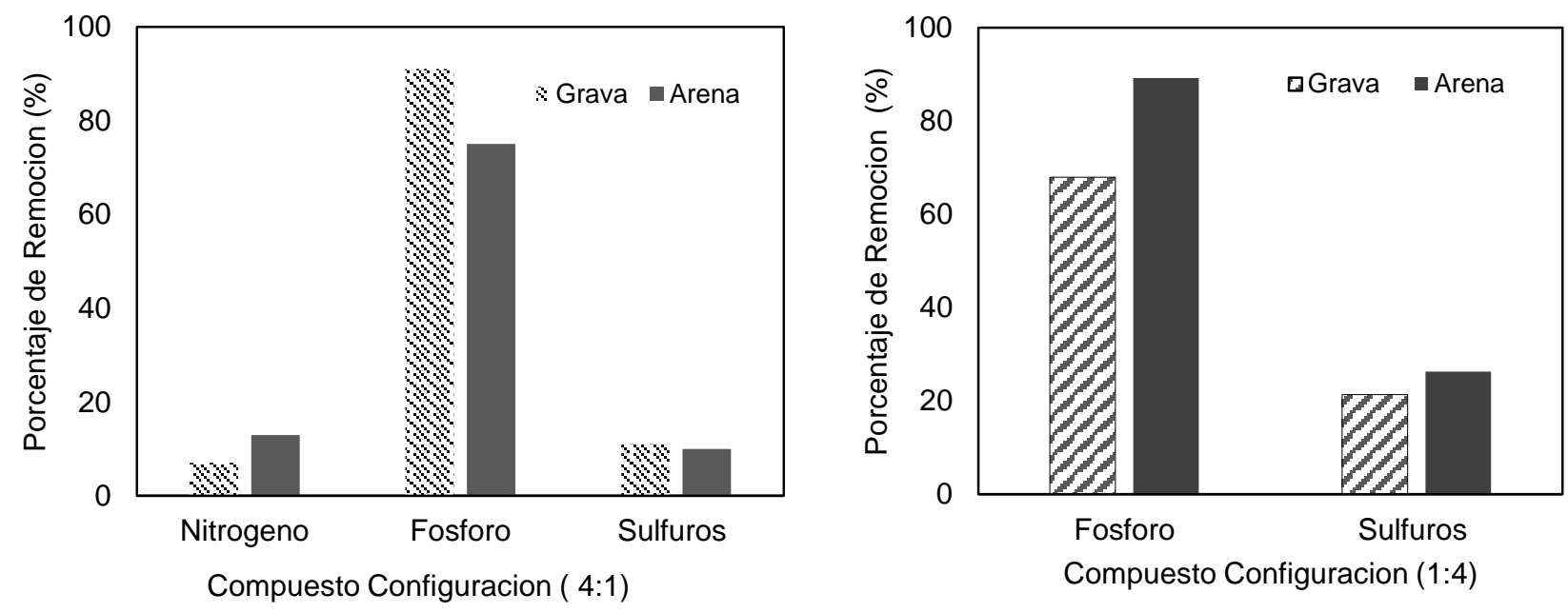

Fig. 3: Remoción de contaminantes en los bioreactores de grava y arena en las configuraciones 4:1 y 1:4.

En ambas configuraciones 4:1 y 1:4 se removió una mayor concentración de fósforo en comparación con los otros contaminantes. En los bioreactores con medio filtrante de grava y configuración $4: 1$ se removió un $91 \%$ de fósforo del agua residual. Esto coincide con el análisis de las condiciones hidráulicas que indicó que el material de grava favoreció la dispersión uniforme del flujo del trazador en el TRH calculado para el bioreactor con configuración 4:1 obteniendo un mayor porcentaje de remoción. En comparación con el análisis hidráulico la configuración 4:1 no presenta zonas muertas lo que favorece la remoción. En la configuración 1:4 la remoción de fósforo más alta se obtuvo en el medio filtrante de arena con $89,22 \%$, siendo afectada por la presencia de zonas muertas.

En comparación con la literatura los resultados obtenidos para la remoción del fósforo en los reactores de grava se asemejan a los presentados en Lee et al., 2009, y Wathugala, et al., 1986, entre $55 \%$ y $99.00 \%$ respectivamente. El reactor con arena utilizado en esta investigación presenta un valor intermedio entre estos.

La remoción de sulfuros obtenida en las dos configuraciones 1:4 y 4:1 de los bioreactores fue baja entre un 10.0 y $26.27 \%$ de remoción en comparación a los otros contaminantes. Estos resultados demuestran que la remoción de sulfuros es limitada y se debe a la tolerancia de la planta $P$. australis. En comparación con estudios realizados por Gonzalías 2007, los resultados en el reactor grava configuración 1:4 fueron similares en remoción de sulfuros. Para un reactor de arena se observaron mejores resultados que la literatura ya que demuestran que la planta $P$. australis puede remover sulfuros a concentraciones de $0.5 \mathrm{mg} / \mathrm{l}$ con $26.27 \%$, pero estos resultados de sulfuros totales se ve influenciado aun por la presencia de sulfatos en el agua residual los cuales tolera más la planta (Gonzalias, 2007, Wu, et al. 2012; Wu, et al. 2011).

\section{Crecimiento del material vegetal}

El crecimiento del material vegetal ( $P$. australis) expuesto a los contaminantes presentes en el agua residual demostró que el desarrollo morfológico de las plantas se vio directamente relacionado con la eficiencia hidráulica de los bioreactores. En la Figura 4 se reporta la magnitud media del incremento de los parámetros evaluados en la vegetación, en ambas configuraciones 1:4 y 4:1. En los resultados se observó que las plantas ubicadas en el bioreactor de grava con configuración 4:1, se presentó un crecimiento más pronunciado de la 
rizosfera (rizomas y raíces) y simultáneamente un crecimiento del tallo. En el reactor de arena la longitud del tallo fue más pronunciada, pero el crecimiento en raíces fue menor. En la configuración 1:4 en el sustrato de grava también presentó mejores resultados en el crecimiento de la raíz y longitud de tallo. Los resultados del crecimiento de $P$. australis fueron directamente relacionados con el comportamiento hidráulico de los reactores, ya que en los reactores con ausencia de zonas muertas y cortos circuitos garantizaron un mayor tiempo de contacto entre la rizosfera de la planta y el contaminante del agua residual, lo que favoreció el desarrollo morfológico de las plantas debido a la abundancia de nutrientes como nitrógeno, fósforo y sulfuros en el medio.
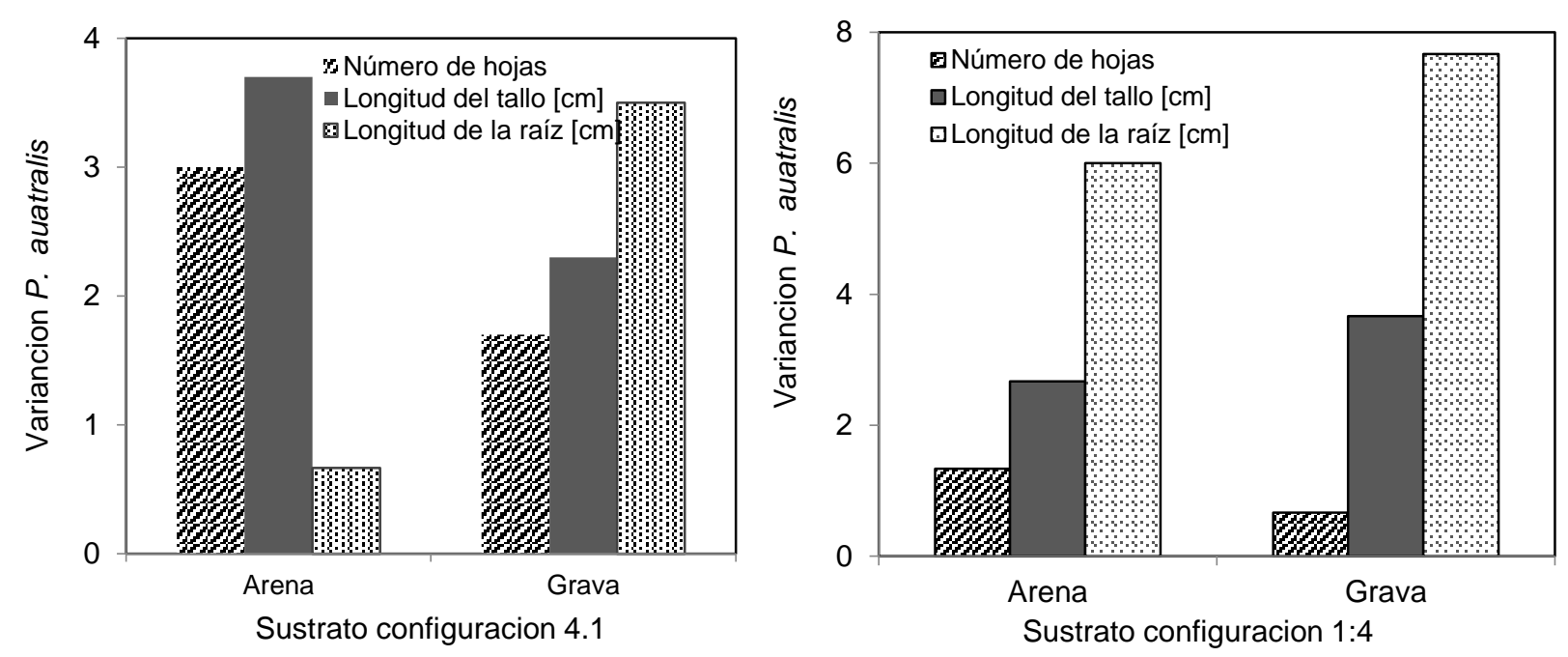

Fig. 4: Crecimiento de raíz, tallo, cantidad de hojas medidas en Pragmites australis en los reactores de grava y arena en las configuraciones $4: 1$ y $1: 4$.

\section{CONCLUSIONES}

Las condiciones hidráulicas óptimas del diseño de los bioreactores, con base en los análisis realizados, para el tratamiento con flujo subsuperficial de agua residual doméstica usando $P$. australis es el reactor con configuración largo:ancho de 4:1 usando grava como material filtrante. Este tipo de reactor contribuyo a mejorar el valor de TRH real, con una mejor eficiencia hidráulica y con la menor proporción de cortos circuitos y zonas muertas, en comparación con los demás bioreactores.

En los reactores con grava independiente de la configuración geométrica empleada, se presentó un crecimiento más pronunciado de la rizosfera (rizomas y raíces) e incremento proporcional de la longitud del tallo. Este desarrollo morfológico de los individuos de $P$. australis en agua residual doméstica establece una relación con la eficiencia hidráulica de los biorreactores. Los mayores porcentajes de remoción de fósforo, nitrógeno y sulfuros utilizando $P$. australis en agua residual doméstica se obtuvieron bajo las condiciones de geometría de 4:1 utilizando grava como sustrato en un tiempo hidráulico de 30 horas.

Para completar los estudios obtenidos en los reactores con una configuración geométrica 4:1 en presencia de grava y la aplicación de $P$. australis como planta fitoremediadora se recomienda realizar un estudio para identificar la microbiota de la rizosfera

\section{AGRADECIMIENTOS}

Los autores agradecen a la Universidad Pontificia Bolivariana Seccional Bucaramanga- Dirección de Investigaciones (DGI) por el soporte financiero a través del proyecto DGI-BI048B y al Laboratorio de Análisis Químico de Aguas Residuales de la Universidad Pontificia Bolivariana-Seccional Bucaramanga

\section{REFERENCIAS}

Ayaz, S.C, Post-treatment and Reuse of Tertiary Treated Wastewater by Constructed Wetlands, doi:10.1016/j.desal.2007.02.110, Desalination. (en línea) 226, 249-255 (2008) 
Al-Dahhan M.H., P.L. Mills, P. Gupta, L. Hana, M. P. Dudukovic, T.M. Leib y J.J. Lerou, Liquid-Phase Tracer Responses in a Cold-flow Counter-current Trayed Bubble Column from Conductivity Probe Measurements, Chem.Eng. Proc. (45) 945-953 (2006)

Carlson M. L., Kowalski K.P., Galbraith D. Assessment of suitable habitat for Phragmites australis(common reed) in the Great Lakes coastal zone. doi: http://dx.doi.org/10.3391/ai.2014.9.1.01

Aqua. Inv. (en linea) 9(1), 1-19 (2014)

Espinosa, C.E, Factibilidad del Diseño de un Humedal de Flujo Subsuperficial para el Tratamiento de Aguas Residuales Municipales de 30.000 Habitantes. Tesis de maestría. Dpto. Ing. Civil. Esc. Colombiana de Ingeniería Julio Garavito. Bogotá, Colombia (2014)

Gonzalias, A. E., P.Kuschk, A. Wiessner, M. Jank, M. Kästner y H. Köser, Treatment of an Artificial Sulphide Containing Wastewater in Subsurface Horizontal Flow Laboratory-Scale Constructed Wetlands, doi:10.1016/j.ecoleng.2007.08.002, Ecol. Eng. (en linea), 31(4), 259-268 (2007)

González, G. M. Efecto en la Variación de la Altura Laminar en la Remoción de Patógenos y Nitrógeno en Humedales Construidos de Flujo Subsuperficial Vertical Tratando aguas Residuales Domésticas en Condiciones Tropicales, Tesis de Maestría en Ecotecnología. Facultad de Ciencias Ambientales. Universidad Tecnológica de Pereira. Pereira, Colombia (2014)

Kadlec R., Wallace S. Treatments Wetlands. $2^{a}$ edición,10-110. CRC Press, Boca Raton Florida, USA. USEPA. (United States Environmental Protection Agency) (En linea) 2009.

http://www.sswm.info/sites/default/files/reference_attachments/KADLEC\%20WALLACE\%202009\%20Treatm ent\%20Wetlands\%202nd\%20Edition_0.pdf

Kalipci E. Investigation of Decontamination Effect of Phragmites australis for Konya Domestic Wastewater Treatment, ISSN 1996-0875, J. Med. Plants Res: 5(29), 6571-6577 (2011)

Lee, C., T. D. Fletcher y G. Sun. Nitrogen Removal in Constructed Wetland Systems, doi:10.1002/elsc.200800049, Eng. Life Sci, (en linea), 9(1) 11-22 (2009)

Naderi Nasrabadi M., Behin Nasrabadi J. Plotting Residence Time Distribution Diagram for a Two-Dimensional Airlift Reactor Using Imagej Software. International Journal of Biological, Ecological and Environmental Sciences (IJBEES) ISSN 2277-43941 (en línea) 181-185, http://www.researchgate.net/publication/255996211_Plotting_residence_time_distribution_diagram_for_a_two-

dimensional_airlift_reactor_using_imageJ_software (2012)

Mastrocicco, M., H. Prommer, L. Pasti, S. Palpacelli y N.Colombani, Evaluation of Saline Tracer Performance During Electrical Conductivity Groundwater Monitoring, Journal of contaminant hydrology, doi:10.1016/j.jconhyd.2011.01.001, (en linea) 123(3-4), 157-66 (2011)

Llano B. A., Cardona, J., Ocampo D. Ríos L. Tratamiento Fisicoquímico de las Aguas Residuales Generadas en el Proceso de Beneficio de Arcillas y Alternativas de Uso de los Lodos Generados en el Proceso, doi: 10.4067/S0718-07642014000300010, Inf. Tecnol. (en línea), 25(3), 73-82 (2014)

Passarini K. C., Gamarra F. M., Vanalle R. y Santana José C. Reutilización de las Aguas Residuales en la Irrigación de Plantas y en la Recuperación de los Suelos. Inf. Tecnol, doi: 10.4067/S071807642012000100007, (en línea), 23(1),57-64 (2012)

Rodríguez M., Miranda R., Olivas R. y Sosa C. Efectos de las Condiciones de Operación Sobre la Biosorción de $\mathrm{Pb}^{2+}, \mathrm{Cd}^{2+} \mathrm{Cr}^{3+}$ en Solución por Saccharomyces cerevisiae Residual. doi:10.1612/inf.tecnol.3977it.07, Inf. Tecnol. (en línea) 19(6), 47-55 (2008)

Sabas, C., Evaluación Hidrodinámica de Humedales Construidos a Escala de Laboratorio, Tesis de Maestría, Facultad de Ciencias Ambientales, Universidad Tecnológica de Pereira, Colombia (2011) http://repositorio.utp.edu.co/dspace/bitstream/11059/2117/1/55149S111.pdf

Valencia, E., I.J. Silva, C.P. Narváez, Sistemas Descentralizados Integrados y Sostenibles para el Tratamiento de Aguas Residuales Domesticas. Revista Ingeniería y Región ( 7) 65-72 (2010) 
Vargas, C. y L .Pardón, Tratamiento de Agua para Consumo Humano Plantas de Filtración Rápida Manual III: Evaluación de Plantas de Tecnología Apropiada. Centro Panamericano de Ingeniería Sanitaria y Ciencias del Ambiente. CEPIS, Lima, Peru. (en línea), 2005.

http://www.bvcooperacion.pe/biblioteca/bitstream/123456789/575/1/BVCI0000575.pdf Acceso: 21 de Junio (2013)

Vera I., I. Ruigómez, E. González, P. Bodelón y J. Rodríguez, Biorreactores de Membrana Anaerobios para Tratamiento de Aguas Residuales Doméstica. Avances en Ciencias e Ingeniería ISSN: 07188706.(en linea), 5(4) 1-15 (2014)

Vymazal, J. y L. Kröpfelová, Nitrogen and Phosphorus Standing Stock in Phalaris arundinacea and Phragmites australis in a Constructed Treatment Wetland: 3-Year Study, doi: 10.1080/03650340701787662 Arch. Agron. Soil Sci., (en linea) 54(3) 297-308 (2008)

Wathugala, A. G., T. Suzuki y Y. Kurihara, Removal of Nitrogen, Phosphorus and Cod from Waste Water Using Sand Filtration System with Phragmites australis, Wat. Research: 21(10) 1217-1224 (1987)

Wiessner, A., K.Z. Rahman, P. Kuschk, M.Kastner, M. Jechorek. Dynamics of sulphur compounds in horizontal sub-surface flow laboratory-scale constructed wetlands treating artificial sewage, doi:10.1016/j.watres.2010.07.044, Wat. Res., 44, 6175-6185 (2010)

Wu, S., Chen Z., Braeckevelt M., Seeger E.M., Dong R., Ka“stner M., Paschke H., Hahn A., Kayser G.,y Kuschk P. Dynamics of Fe(II), Sulphur and Phosphate in Pilot-scale Constructed Wetlands Treating a Sulphate-rich Chlorinated Hydrocarbon Contaminated Groundwater, doi:10.1016/j.watres.2012.01.011, Wat. Res. 46, 1923 -1932 (2012)

Wu S., Jeschke, C., Dong R., Paschke H., Kuschk P., Knoller K. Sulfur transformations in pilot-scale constructed wetland treating high sulfate-containing contaminated groundwater: A stable isotope assessment, doi:10.1016/j.watres.2011.10.008. Wat. Res., (en línea) ,45, 6688-6698, (2011)

Zataray, J. P., S. Chong, R. Radilla, R. Ramos, F. T., Wakida, E. Vélez, J.E. Castillo y J.G. Rodríguez, Diseño de un Sistema de Humedales de Flujo Subsuperficial (SSF) para el Tratamiento Terciario del Agua del Río Tecate, una Alternativa de Reusó. Universidad Autónoma de Baja California, México, (2006) http://www.uaemex.mx/Red_Ambientales/docs/memorias/Extenso/TA/EO/TAO-68.pdf Acceso: 2 de Octubre (2012) 
